\title{
Heparanase induces tissue factor pathway inhibitor expression and extracellular accumulation in endothelial and tumor cells
}

\author{
Yona Nadir ${ }^{1,2}$, Benjamin Brenner², Sveta Gingis-Velitski', Flonia Levy-Adam', Neta Ilan', Eyal Zcharia ${ }^{3}$ Erez Nadir', \\ Israel Vlodavsky' \\ 'Cancer and Vascular Biology Research Center, The Bruce Rappaport Faculty of Medicine, Technion, Haifa, Israel; ${ }^{2}$ Thrombosis and Hemostasis \\ Unit, Department of Hematology, Rambam Medical Center, Haifa, Israel; ${ }^{3}$ Department of Oncology, Hadassah-Hebrew University Medical \\ Center, Jerusalem, Israel; ${ }^{4}$ Department of Neonatology, Hillel Yaffe Medical Center, Hadera, Israel
}

\section{Summary}

Heparanase activity is implicated in cell invasion, tumor metastasis and angiogenesis. Recently, we have reported that heparanase stimulates tissue factor (TF) expression in endothelial and cancer cells, resulting in elevation of coagulation activity. We hypothesized that heparanase regulates other coagulation modulators, and examined the expression and localization of tissue factor pathway inhibitor (TFPI) following heparanase over-expression or exogenous addition. Primary human umbilical vein endothelial cells (HUVEC) and human tumor-derived cell lines were incubated with heparanase, or were stably transfected with heparanase gene-constructs, and TFPI expression and secretion were examined. Heparanase over-expression or exogenous addition stimulated TFPI expression by $2-3$ folds. TFPI accumulation in the cell culture medium exceeded in magnitude the observed induction ofTFPI gene transcription reaching 5- to

\section{Keywords}

TFPI, heparanase, coagulation, endothelial cells, tumor 6-fold increase. Extracellular accumulation of TFPI was evident already $60 \mathrm{~min}$ following heparanase addition, prior to TFPI protein induction, and correlated with increased coagulation activity. This effect was found to be independent of heparanase enzymatic activity and interaction with heparan-sulfate, and correlated with reduced TFPI levels on the cell surface. Data were verified in heparanase transgenic mice tissues and plasma. Interaction between heparanase and TFPI was evident by co-immunoprecipitation. Interaction of heparanase with TFPI resulted in its displacement from the surface of the vascular endothelium and in increased pro-coagulant activity. Thus, heparanase facilitates blood coagulation on the cell surface by two independent mechanisms: dissociation ofTFPI from the vascular surface short after local elevation of heparanase levels, and subsequent induction of TF expression.

\section{Introduction}

Heparanase is an endo- $\beta$-D-glucuronidase that is capable of cleaving heparan sulfate (HS) side chains of heparan sulfate proteoglycans (HSPG) on cell surfaces and the extracellular matrix, activity that is strongly implicated in cell dissemination associated with tumor metastasis and angiogenesis $(1,2)$. Indeed, heparanase up regulation has been documented in an increasing number of primary human tumors $(2,3)$, including pancreas $(4$, 5 ), bladder (6), gastric (7, 8), cervical (9) and colorectal (10) tumors, collectively providing a clinical relevance for this enzyme, and positioning heparanase as a valid target for the development of anti cancer drugs (2). Apart from its well characterized enzymatic activity, heparanase was also noted to exert enzymatic-in- dependent functions. Among these is the induction of Akt/PKB phosphorylation noted in endothelial and tumor derived cells, facilitating cell motility (11), and Src-dependent VEGF induction (12), providing, among other mechanisms (13), a molecular basis for the pro-angiogenic feature of the protein. In addition, we recently reported that heparanase induces expression of tissue factor (TF) by endothelial and tumor-derived cells, leading to enhanced coagulation (14). TF induction was evident in stably transfected cells over expressing heparanase, as well as upon exogenous addition of the heparanase protein. It required no enzymatic activity and appeared to involve the $\mathrm{p} 38$ pathway (14), previously noted to be activated by heparanase (12). Furthermore, a direct correlation between heparanase and $\mathrm{TF}$ expression levels was noted in leukemia patients, clearly supporting a clinical rel-

Correspondence to: 
evance of this finding (14). Given the importance of blood coagulation in hemostasis and its tight regulation, we hypothesized that heparanase not only induces TF expression, but is also involved in the regulation of additional factors participating in the coagulation machinery. We examined here the possible involvement of heparanase in the regulation of tissue factor pathway inhibitor (TFPI), a potent, endogenous inhibitor of factor Xa and factor VIIa-TF complex. TFPI is expressed by endothelial cells and other cells of the vascular wall, contributing to the nonthrombogenic properties of the endothelial cell surface. We provide evidence that heparanase over-expression or exogenous addition induces 2- to 3-fold increase in TFPI expression, evident by immunoblotting, ELISA, and real-time PCR analyses. Similarly, heparanase stimulated accumulation of TFPI in the cell culture medium. Extracellular accumulation exceeded, however, in magnitude the observed increase in TFPI at the protein level, and appeared to be independent of HS and heparanase enzymatic activity. Instead, we have found a physical interaction between heparanase and TFPI, suggesting a mechanism by which secreted heparanase interacts with TFPI on the cell surface, leading to dissociation of TFPI from the plasma membrane and increased coagulation activity, thus further supporting the local pro-thrombotic function of heparanase.

\section{Materials and methods}

\section{Cell culture and transfection}

U87-MG human glioma, MDA-MB-435 human breast carcinoma, HT-29 human colon carcinoma, and HEK-293 cells were purchased from the American Type Culture Collection (ATCC). Cells were grown in Dulbecco's modified Eagle's medium (Biological Industries, Beit Haemek, Israel) supplemented with 10\% fetal calf serum and antibiotics. Human umbilical vein endothelial cells (HUVEC) were kindly provided by Dr. Neomi Lanir (Rambam Medical Center, Israel), and grown in gelatin-coated flasks, essentially as described (11). Sub-confluent U87, MDA435, HT-29 and HEK-293 cells were stably transfected with the human heparanase cDNA cloned into the pcDNA3 plasmid, using FuGENE 6 reagent according to the manufacturer's (Roche Applied Science, Indianapolis, IN, USA) instructions, as described (15-17). Transfection proceeded for 48 hours, followed by selection with G418 (Invitrogen, Carlsbad, CA, USA) for two weeks. Stable transfectant pools were further expanded and analyzed. Modified species of heparanase cloned into the pcDNA3 vector were used: heparanase gene construct mutated at $\mathrm{Glu}^{225}$ and $\mathrm{Glu}^{343}$, critical for the enzyme catalytic activity (17, 18), heparanase gene construct deleted for the N-terminal 35 amino acids $\left(\mathrm{Met}^{1}-\mathrm{Ala}^{35}\right)$ that comprises the protein signal peptide (12) and $65 \Delta 10$ variant including a deletion of an identified heparin binding domain $\left(\mathrm{Gln}^{270}-\mathrm{Lys}^{280}\right)$ (19).

\section{Antibodies and reagents}

Polyclonal antibody 1453 was raised in rabbits against the entire $65 \mathrm{kDa}$ heparanase precursor isolated from the conditioned medium of heparanase-transfected HEK-293 cells. The antibody was affinity purified on immobilized bacterially expressed 50 $\mathrm{kDa}$ heparanase GST fusion protein (17). Anti-heparanase monoclonal antibody 130 was kindly provided by InSight Phar- maceuticals (Rehovot, Israel). Monoclonal (ID 4903) and polyclonal anti-human TFPI (ID 4901) antibodies were purchased from American Diagnostica (Stanford, CT, USA) and monoclonal anti-actin antibody was purchased from Sigma (St. Louis, MO, USA). Single chain GS3 active heparanase gene construct, comprised of the $8 \mathrm{kDa}$ and $50 \mathrm{kDa}$ heparanase heterodimer $(8+50)$, was kindly provided by Dr. Christian Steinkuhler (IRMB/Merck Research Laboratories, Pomezia, Italy) (20), and the protein was purified from the conditioned medium of baculovirus infected cells (14). GS3 active heparanase was assayed for the presence of bacterial endotoxin by Biological Industries (Beit Haemek, Israel), using the gel-clot technique (Limulus amebocyte lysate LAL test) and was found to contain $<10 \mathrm{pg} / \mathrm{ml}$ endotoxin. Recombinant human TFPI (ID 4900PC) was purchased from American Diagnostica (Stanford, CT, USA). Flavobacterium heparinum heparinase II was kindly provided by IBEX Technologies (Montreal, Canada) and Trichloroacetic Acid (TCA) was purchased from Sigma (St. Louis, MO, USA).

\section{Culture media proteins precipitation}

One hundred $\mu \mathrm{l}$ of $20 \%$ TCA was added to $1 \mathrm{ml}$ serum free culture media. The sample was incubated for $15 \mathrm{~min}$ on ice and was then spun for $15 \mathrm{~min}$ at $4^{\circ} \mathrm{C}$. The supernatant was carefully removed. $200 \mu \mathrm{l}$ of cold acetone was added and spun for five minutes. This step was repeated twice. The supernatant was removed and the pellet was left about $10 \mathrm{~min}$ to dry at $37^{\circ} \mathrm{C}$. The pellet was resuspended in phosphate buffered saline (PBS) and samples were normalized for total protein levels in the cells from which the media were derived.

\section{SDS-PAGE and immunoblot analysis}

Cell extracts and tissues were prepared using a lysis buffer consisting of $50 \mathrm{mM}$ Tris-HCl, pH 7.4, $150 \mathrm{mM} \mathrm{NaCl}, 0.5 \%$ Triton X-100, supplemented with protease inhibitors (Roche, Nutley, NJ, USA). Equal amounts of protein were subjected to $10 \%$ SDS-polyacrylamide gel electrophoresis (PAGE) and transferred to polyvinylidene fluoride membrane (BioRad, Maylands, CA, USA). The membrane was probed with the appropriate antibody followed by horseradish peroxidase (HRP)-conjugated secondary antibody (Jackson ImmunoResearch, West Grove, PA, USA) and chemiluminescence substrate (Pierce, Rockford, IL, USA), as described (14).

\section{TFPI ELISA}

TFPI levels were studied by IMUBIND ${ }^{\circledR}$ Total TFPI ELISA Kit (American Diagnostica, Stanford, CT, USA). TFPI levels in lysates and serum free medium of $5 \times 10^{5}$ cells $/ \mathrm{ml} \mathrm{U87} \mathrm{(overnight}$ incubation) and HUVEC ( $6 \mathrm{~h}$ incubation) cells were analyzed. Equal amounts of cell lysate protein were used and medium samples were normalized for total protein in the cells from which the media were derived. Lysates were diluted 1:50 in sample buffer of the Kit. The ELISA employs rabbit anti-human TFPI polyclonal antibody as a capture antibody. Medium and diluted lysate samples were incubated in microwells precoated with the capture antibody. TFPI was detected using a monoclonal antibody specific for the Kunitz domain 1 of TFPI. Next, streptavidin conjugated HRP was bound to the secondary antibody. Added TMB substrate reacted with HRP, yielding a blue colored 
solution. The reaction was terminated by the addition of $0.5 \mathrm{M}$ sulfuric acid, changing the color of the reaction mixture to yellow. TFPI levels were determined by measuring the absorbance at $450 \mathrm{~nm}$.

\section{TFPI activity assay}

TFPI anticoagulant activity was analyzed by Actichrome ${ }^{\circledR}$ TFPI activity assay (American Diagnostica, Stanford, CT, USA). $\operatorname{HUVEC}\left(5 \times 10^{2}\right.$ cells/well $)$ were seeded into 96-well plate and allowed to grow overnight. Cells were then washed twice with the assay reaction buffer and further analyzed. TFPI anticoagulant activity in serum free medium of $5 \times 10^{5}$ cells $/ \mathrm{ml}$ U87 (overnight incubation) and HUVEC ( $6 \mathrm{~h}$ incubation) cells, was also analyzed. Next, cells or medium were incubated with TF/factor VIIa and factor X. The amount of factor Xa generated was measured by its ability to cleave Spectrozyme Xa, a chromogenic substrate for factor Xa. Cleavage released a chromophore into the reaction solution which was quantified by measuring the absorbance at $405 \mathrm{~nm}$. Medium samples were normalized for total protein levels of the cells from which the media were derived.

\section{RNA isolation and CDNA preparation}

Total RNA was isolated by TRIzol reagent (Life Technologies, Gaithersburg, MD, USA). RNA ( $1 \mu \mathrm{g})$ was resolved by $1 \%$ agarose gel electrophoresis and was visualized by ethidium bromide staining. The cDNA preparation was obtained using oligo(dT) priming and reverse transcriptase enzyme, as described (14).

\section{Real time RT-PCR}

RT-PCR was performed using the ABI PRISM 7700 Sequence Detection System, applying TaqMan Universal PCR Master Mix, Assays-on-Demand Gene Expression probes (Applied Biosystems, Foster City, CA, USA) and cDNA, for the PCR step. The following conditions were applied for TFPI and L-19 (control gene) PCR amplification: 40 cycles of $50^{\circ} \mathrm{C}$ for two minutes, and of $95^{\circ} \mathrm{C}$ for 10 minutes.

Figure I: Heparanase induces TFPI expression and extracellular accumulation. A-D) Stable transfection. A) Lysate samples prepared from heparanase transfected (Hepa) MDA- 435 breast carcinoma (left panels) and U87 glioma cells (right panels), and their corresponding mock transfected control cells (Con) were subjected to immunoblot analysis with anti-TFPI (middle panels) and anti-actin (lower panels) antibodies. Medium conditioned by control and heparanase transfected cells was similarly immunoblotted with anti TFPI antibody (upper panels). B) Relative assessment of TFPI using TFPI ELISA and activity assay $(n=3$, mean \pm SD). Heparanase transfected (Hepa) U87 glioma cells and their corresponding mock transfected control cells (Con) were analyzed for TFPI levels in the lysates (left) and medium (middle). TFPI anticoagulant activity was measured in the medium (right). While a 2 -fold increase in TFPI levels was observed in Hepa transfected cell lysates $(p<0.01)$, a 5- to 6-fold increase in TFPI levels was noted in the medium of these cells compared to control cells $(p<0.005)$. No statistically significant difference was found in TFPI activity determined in the medium of these cells. C) Real time-PCR. Total RNA was extracted from heparanase (Hepa)- and mock (Con)- transfected MDA-435 and U87 glioma cells. TFPI expression was analyzed by real-time PCR analysis $(n=3$, mean \pm SD). An increase in TFPI mRNA level was observed in MDA-435 cells $(p<0.0 I)$ and $U 87$ cells $(p<0.005)$.

\section{Heparanase transgenic mice}

Transgenic mouse model in which human heparanase expression is driven by the $\beta$-actin promotor, thus enabling high levels of expression in essentially all tissues (21), was utilized.

\section{FACS analysis}

Flow-cytometry analysis was performed essentially as described (14). Briefly, cells $\left(1 \times 10^{6}\right)$ were incubated for $15 \mathrm{~min}$ at $4^{\circ} \mathrm{C}$ with polyclonal non-conjugated rabbit anti-human TFPI (American Diagnostica, Stanford, CT, USA; 1:50). Non-conjugated normal rabbit IgG (Santa Cruz Biotechnology, Santa Cruz, CA, USA; 1: 50) was employed as control. FITC anti-rabbit antibody (Santa Cruz Biotechnology, Santa Cruz, CA, USA; 1:100) was

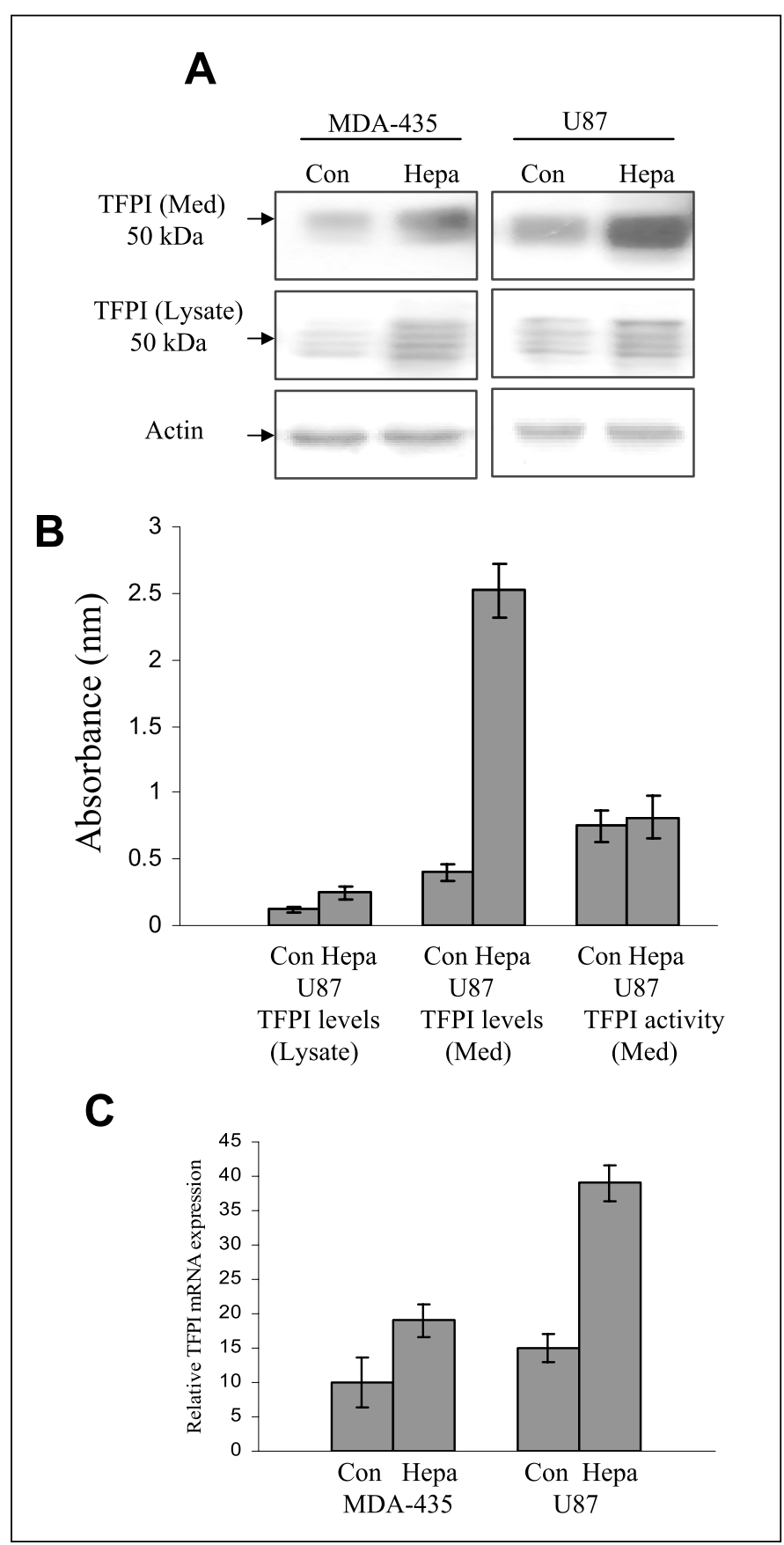


D

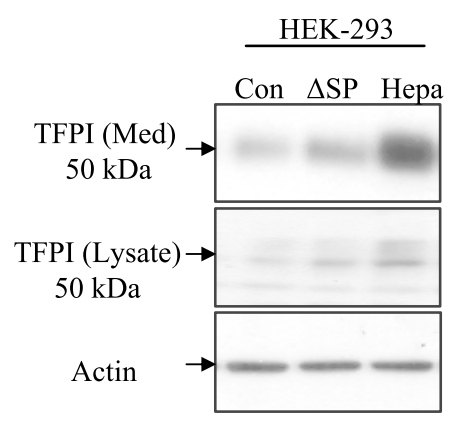

$\mathbf{F}$
E

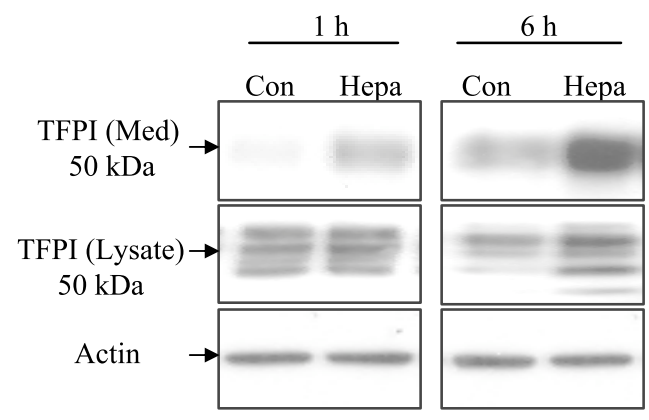

G
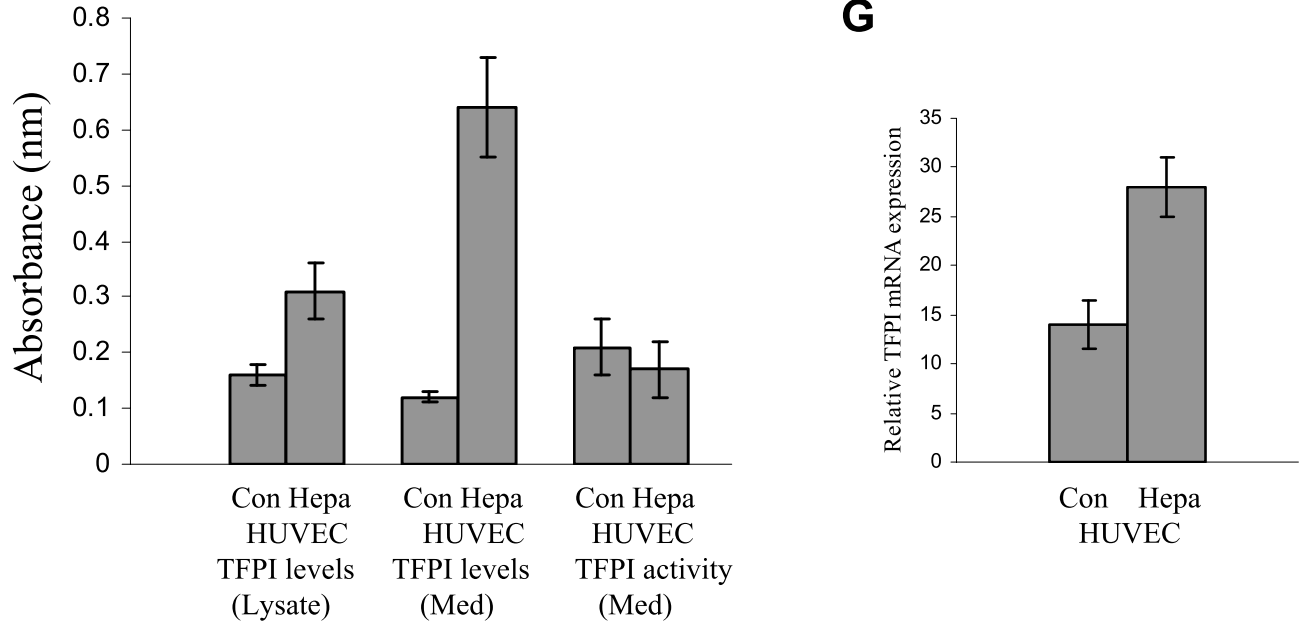

Figure I (continued): Heparanase induces TFPI expression and extracellular accumulation. D) HEK-293 cells stably transfected with wild type (Hepa), or a gene construct in which the heparanase signal peptide was deleted ( $\triangle \mathrm{SP}$ ), or control mock transfected cells (Con) were similarly analyzed for TFPI expression (middle panel) and extracellular accumulation (upper panel). E,F,G. Exogenous addition. E) Immunoblot analysis. Primary endothelial cells (HUVEC) were left untreated (Con) or incubated with GS3 active heparanase (Hepa; I $\mu \mathrm{g} / \mathrm{ml})$ in serum free medium for $\mathrm{I} h$ and $6 \mathrm{~h}$. TFPI expression and extracellular accumulation were evaluated by immunoblotting, as previously described. F) Relative assessment of TFPI using TFPI ELISA and activity assay $(n=3$, mean $\pm S D)$. GS3 active heparanase $(\mathrm{Hepa}$; $\mu \mathrm{g} / \mathrm{ml})$ was ex- ogenously added to HUVEC in serum free medium for $6 \mathrm{~h}$. TFPI in lysates (left) and medium (middle) were assessed by ELISA. TFPI anticoagulant activity was measured in the medium (right). While a 2 -fold increase in TFPI was observed in Hepa transfected cell lysates $(p<0.01)$, a 6 -fold increase in TFPI levels was noted in the medium of these cells compared to control cells $(p<0.005)$. No statistically significant difference was found in TFPI activity determined in the medium of these cells, similar to the results with U87 glioma cells. G) Real time PCR. Total RNA was extracted following $6 \mathrm{~h}$ incubation of HUVEC with active heparanase, as described in E. TFPI expression was then evaluated by real time RT-PCR $(n=3$, mean $\pm S D)$. Two-fold increase in TFPI mRNA level was observed following heparanase addition $(p<0.0 \mathrm{I})$.

incubated with the quenching buffer and sodium cyanoborohydride at $20^{\circ} \mathrm{C}$ for 30 minutes. Next, the gel was washed four times with wash solution, once with elution buffer, and twice with coupling buffer. Recombinant human TFPI $(0.5 \mu \mathrm{g})$ and GS3 active heparanase $(0.5 \mu \mathrm{g})$, or serum free medium (about $400 \mu \mathrm{l}$, samples were normalized for total protein levels of the cells from which the media were derived) were added to the gel and incubated for two hours at $4^{0} \mathrm{C}$. The gel was washed four times with coupling buffer and bound proteins were eluted with elution buffer (pH 2.5) neutralized by $1 \mathrm{M}$ Tris- $\mathrm{HCl}, \mathrm{pH} 9.5$ and subjected to immunoblot analysis. Polyclonal anti-TFPI and monoclonal anti-heparanase antibodies were used to detect the respective coupled protein. Irrelevant, anti-GST polyclonal antibody $(100 \mu \mathrm{g})$ and uncoupled beads were used as controls. four hours. The gel was then washed with quenching buffer and

The interaction between TFPI and heparanase was analyzed by co-immunoprecipitation (co-IP). The ProFound ${ }^{\text {Tw }}$ Co-Immunoprecipitation Kit in which the antibody is coupled to gel support was employed according to the manufacturer's (Pierce, Rockford, IL, USA) instructions. Briefly, coupling gel was washed with coupling buffer. Polyclonal anti TFPI or polyclonal anti heparanase (1453) antibodies $(100 \mu \mathrm{g})$ and $5 \mathrm{M}$ sodium cyanoborohydride, were added to the gel support and incubated at $4^{0} \mathrm{C}$ for 


\section{Statistical analysis}

Data were evaluated by SPSS software for Windows version 13.0 (SPSS Inc., Chicago, IL, USA). Student's t-test for independent samples was used. Values are reported as mean $\pm \mathrm{SD}$. $\mathrm{P}<0.05$ was considered significant. All experiments were repeated at least three times, with similar results.

\section{Results}

\section{Heparanase induces TFPI expression and extracellular accumulation}

We have recently reported that heparanase over expression or exogenous addition enhances the expression of the pro-coagulant $\mathrm{TF}$, resulting in elevated coagulation activity in endothelial and tumor-derived cells (14). Given the importance of homeostasis regulation, we hypothesized that coagulation factors other than TF are affected by heparanase, and suspected that TFPI is also regulated by heparanase. In order to examine this hypothesis, control mock transfected (Con) and heparanase transfected (Hepa) MDA-435 breast carcinoma (Fig. 1A) and U87 glioma (Fig. 1A) cell lysates were subjected to immunoblotting with anti-TFPI antibody. Control mock transfected U87 glioma and MDA-435 breast carcinoma cells expressed detectable levels of TFPI (Fig. 1A, middle panels) while heparanase over expression resulted in increased TFPI levels (Fig. 1A, middle panels). ELISA determination revealed a nearly 2 -fold increase in TFPI levels in U87 cells over-expressing heparanase (Hepa) (Fig. 1B, left). These results were substantiated by real-time PCR analysis, revealing 2- to 3-fold increase in TFPI mRNA levels in heparanase transfected MDA-435 and U87 cells (Fig. 1C), indicating that heparanase induces TFPI gene transcription. Similarly, elevated levels of TFPI were observed in the culture medium of heparanase transfected cells (Fig. 1A, upper panels). Moreover, elevation of TFPI in the medium exceeded the observed induction in gene transcription, as best exemplified in heparanase transfected U87 (Fig. 1A) and HEK-293 (Fig. 1D) cells. ELISA determination revealed that while heparanase over expression resulted in a 2- to 3-fold increase in TFPI expression by U87 cells (Fig. 1B, left), TFPI levels in the culture medium were increased 5- to 6-fold (Fig. 1B, middle). Notably, the increased levels of TFPI in the medium had no effect on TFPI activity (Fig. 1B, right). In addition, over-expression of a non-secretable heparanase gene construct, in which the protein signal peptide was deleted $(\triangle \mathrm{SP})(12)$, failed to induce TFPI expression and its extracellular accumulation (Fig. 1D). This led us to suspect that extracellular accumulation of TFPI is induced by the secreted heparanase protein. In order to ascertain this possibility, human umbilical vein endothelial cells (HUVEC) were left untreated as control (Con) or incubated with recombinant active $50+8 \mathrm{kDa}$ heparanase (GS3) heterodimer (Hepa, $1 \mu \mathrm{g} / \mathrm{ml}$ ) in serum free medium for $1 \mathrm{~h}$ and $6 \mathrm{~h}$. Cell lysates and culture media were then subjected to immunoblot analysis applying anti TFPI antibody. As demonstrated in Figure 1E, elevated levels of TFPI were detected in the cell culture medium already $1 \mathrm{~h}$ following heparanase addition (Fig. 1E, upper panel), without a noticeable change in the intracellular levels of TFPI (Fig. 1E, middle panel). After $6 \mathrm{~h}$ incubation, TFPI levels were increased both in the cell lysate and culture medium (Fig. 1E, middle and upper panels), with extra-

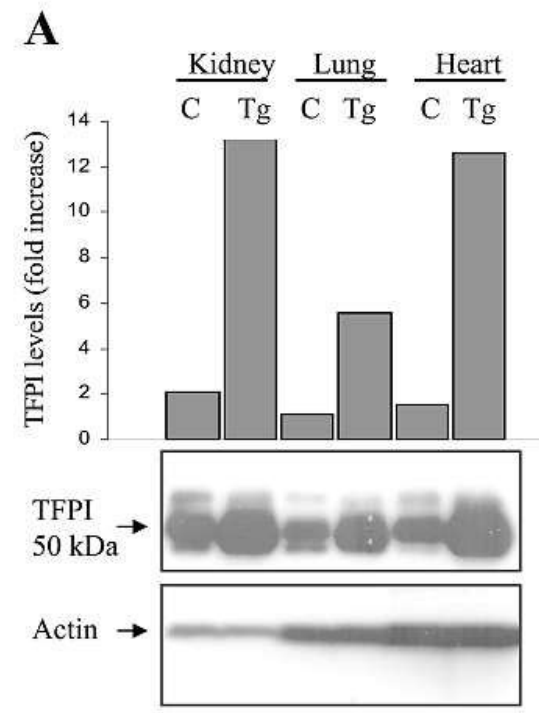

\section{B}

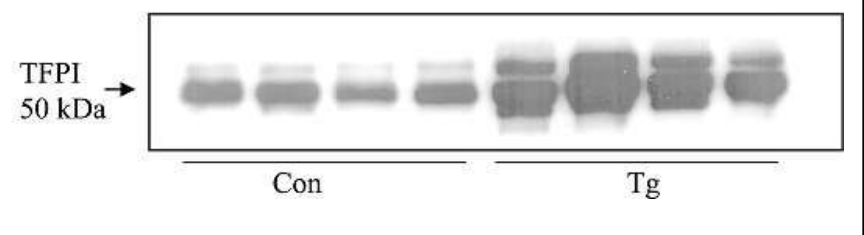

Figure 2: Elevated TFPI levels in heparanase transgenic mice. A) Tissue extracts from heparanase transgenic mice $(\mathrm{Tg})$ and littermate control (C) mice were subjected to immunoblotting with monoclonal anti-TFPI antibody. Densitometric analysis of TFPI levels in each pair of tissues is presented (upper panel). Equal protein loading for each tissue was confirmed by anti-actin antibodies (lower panel). TFPI protein expression in heparanase transgenic mice was higher than in control mice. B) Immunoblot analysis of TFPI in the plasma of control (Con, left) and heparanase transgenic $(\mathrm{Tg}$, right) mice.

cellular accumulation exceeding the increase in protein synthesis. ELISA analysis indicated that TFPI levels were elevated 1.9-fold (Fig. 1F, left) due, in part, to a transcriptional effect evident by a 2 -fold increase in TFPI mRNA revealed by real time PCR analysis (Fig. 1G). In contrast, TFPI levels in the culture medium were increased 6-fold following heparanase addition (Fig. 1F, middle), with no effect on TFPI activity in the medium (Fig. 1F, right). These results are similar in magnitude and effect to TFPI elevation obtained in stably transfected cells (Fig. 1A-D). These results suggest a dual effect of heparanase, inducing TFPI gene transcription and even more so eliciting its extracellular accumulation.

\section{TFPI expression and circulating levels are elevated in heparanase transgenic mice}

In order to further evaluate the ability of heparanase to regulate TFPI expression and extracellular accumulation, we utilized a transgenic mouse model in which human heparanase expression is driven by the $\beta$-actin promoter, thus enabling high levels of expression in most tissues (21). Lysate samples prepared from kidney, lung and heart tissues of human heparanase transgenic ( $\mathrm{Tg}$ ) 


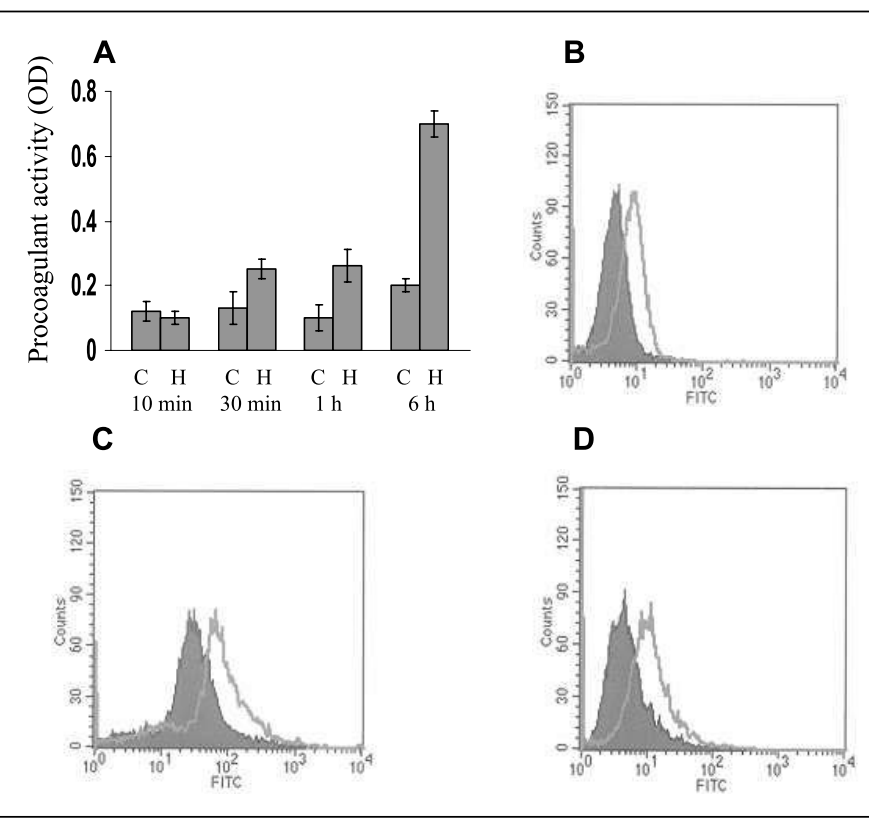

Figure 3: Heparanase induces coagulation and release of TFPI from HUVEC surface. A) Coagulation. HUVEC were left untreated (C) or incubated with heparanase $(\mathrm{H}, \mathrm{I} \mu \mathrm{g} / \mathrm{ml})$ for the indicated time. Coagulation assay was then performed, as described in Materials and methods. The mean value \pm SD of three independent experiments is shown. Note two-fold increase in coagulation activity already after 30 min and I $h$ of incubation with heparanase $(p<0.0 I)$. B-D. FACS analysis. B) Exogenous addition. HUVEC were left untreated as control or incubated with heparanase $(\mathrm{I} \mu \mathrm{g} / \mathrm{ml})$ for $6 \mathrm{~h}$. Heparanase was then washed, and cell-surface TFPI was examined by FACS. Note, reduced TFPI levels on the cell surface following heparanase addition (filled curve). C-D. Stably transfected cells. Cell surface TFPI was examined in heparanase (filled curve) and mock (empty curve) transfected HEK-293 (C) and MDA-435 (D) cells.

and control (Con) mice were subjected to TFPI immunoblot analysis. As shown in Figure 2A, TFPI expression was significantly stimulated in tissues derived from the heparanase transgenic ( $\mathrm{Tg}$ ) vs. control (C) mice. Moreover, plasma levels of TFPI were markedly increased in the heparanase transgenic mice (Fig. 2B), further supporting the in vitro findings (Fig. 1).

\section{Increased coagulation activity in response to heparanase addition}

In order to explore the biological significance of TFPI induction by heparanase, we evaluated TF-dependent coagulation activity in HUVEC incubated with or without heparanase. As shown in Figure 3A, a 2-fold increase in coagulation activity was observed already $30 \mathrm{~min}$ following the addition of recombinant active heparanase and further induction of activity was noted at $6 \mathrm{~h}$, changes that were statistically significant $(\mathrm{p}=0.01$ and 0.005 , respectively). While elevated coagulation activity at late time points $(6 \mathrm{~h})$ is likely to be due to heparanase-induced increase in TF levels (14), elevation of coagulation activity at $30 \mathrm{~min}$ occurred prior to TFPI (Fig. 1G) or TF (14) protein induction. These results raise the possibility that heparanase addition reduces TFPI levels on the cell surface, in agreement with its accumulation extracellularly. We approached this possibility by examining surface localization of TFPI by flow-cytometry. As demonstrated in Figure 3B, cell surface expression of TFPI is significantly decreased following the addition of heparanase. Similar results were obtained by FACS analysis of mock vs. heparanase transfected HEK-293 (Fig. 3C) and MDA- 435 (Fig. 3D) cells.

\section{TFPI release by heparanase is independent of enzymatic activity and HS}

TFPI is constitutively expressed by endothelial cells, as well as other cells of the vessel wall (i.e. smooth muscle cells, cardiomyocytes). Circumstantial evidence suggests that TFPI may be bound to HS on the cell surface (22). We therefore suspected that accumulation of TFPI extracellularly may be due to cleavage of HS by heparanase and the removal of TFPI from the cell surface. Surprisingly, however, the infection of U87 cells with lenti virus vector expressing mutated inactive heparanase resulted in extracellular accumulation of TFPI to a magnitude comparable to the active enzyme (Fig. 4A), suggesting an enzymatic activity-independent effect of heparanase. Heparanase may compete with TFPI and dissociate it from the plasma membrane, since both TFPI and heparanase possibly interact with HS $(15,22)$. In order to examine this possibility, we first used HT-29 colon carcinoma cells reported to express perlecan, a secreted HSPG, but apparently no other proteoglycans (23). Indeed, heparanase uptake by HT-29 cells is significantly attenuated, similar to heparanase uptake by HS-deficient CHO-745 cells (15). Exogenous addition (Ex), or stable expression of heparanase by transfected (Tr) HT29 cells resulted in extracellular accumulation of TFPI (Fig. 4B), comparable in magnitude to cells that express normal levels of HSPG (i.e. MDA-435 and HEK-293 cells, Fig. 1), suggesting HS-independent release of TFPI by heparanase. Moreover, stable transfection of HEK-293 cells with heparanase gene construct deleted for its heparin-binding domain (65 $\Delta 10)$ (19), resulted in TFPI release indistinguishable from that induced by the wild type enzyme (Fig. 4C). We further utilized an enzymatic approach and subjected U87 cells to bacterial heparinase II in order to cleave cell surface HS chains. This treatment resulted in a marked elevation of TFPI levels in the culture medium compared with control untreated cells (Fig. 4D, upper panel), thus further indicating TFPI association with membrane HSPG. Control and heparinase II treated cells were subsequently washed, and incubated with recombinant heparanase for 1 hour. Interestingly, accumulation of TFPI in the culture medium of heparinase IItreated cells was indistinguishable from control cells that were not subjected to heparinase II (Fig. 4D, second panel). Collectively, these results suggest that TFPI release by heparanase is not due to competition of heparanase and TFPI for HS.

\section{Heparanase directly interacts with TFPI}

Next, we sought an alternative explanation for the observed extracellular accumulation of TFPI in response to heparanase. We rationalized that heparanase interacts with TFPI on the cell surface, leading to dissociation of TFPI from the complex TFPIHS-FXa-FVIIa and thereby enhances TF activity on the cell surface (Fig. 3). In order to examine this possibility, overnight incubated serum free medium of control mock transfected (Con) and heparanase transfected (Hepa) U87 cells $\left(1 \times 10^{6} / \mathrm{ml}\right)$ were incubated with immobilized polyclonal anti-TFPI antibody. The 

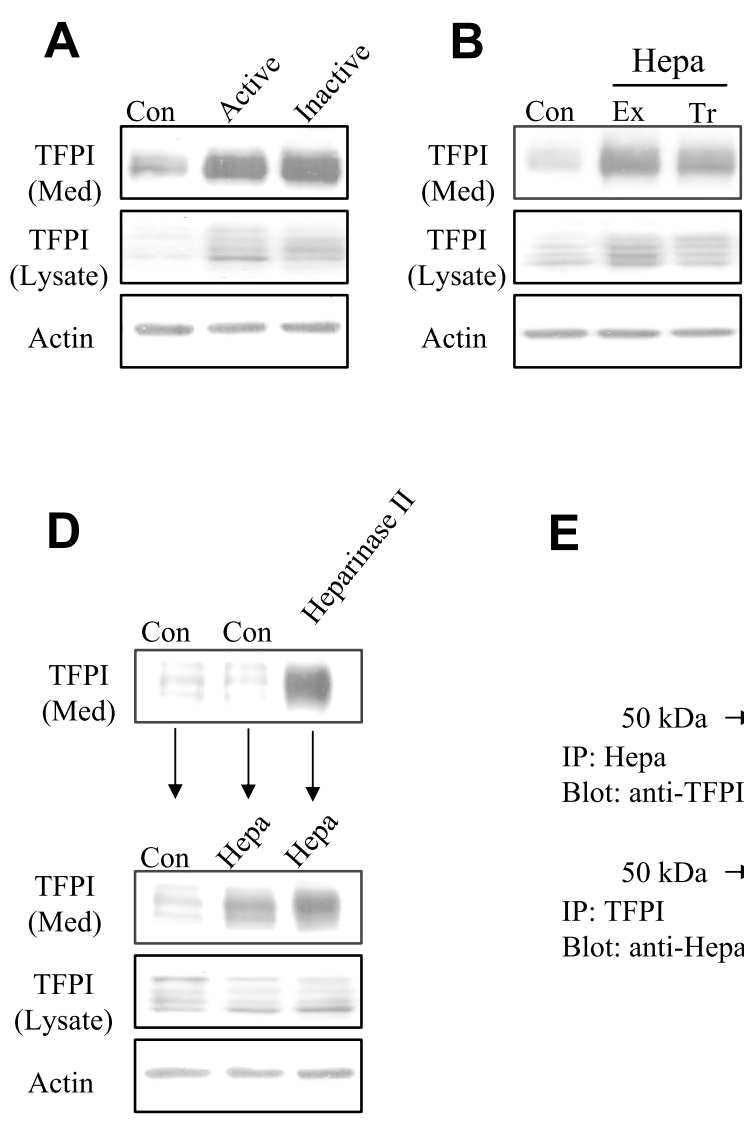

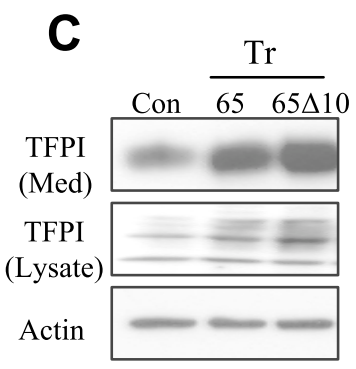

Figure 4: Heparanase-induced TFPI release is independent of enzymatic activity and HS. A) U87 cells were infected with control empty lenti virus, or a viral vector expressing active or inactive heparanase. Cell conditioned medium and lysate samples were subjected to immunoblot analysis with anti TFPI and anti-actin antibodies. Note enhanced TFPI accumulation in the culture medium of cells infected with enzymatically inactive heparanase. B) HT-29 cells were incubated with active heparanase (GS3, I $\mu \mathrm{g} / \mathrm{ml}$, Ex) or stably transfected with full length heparanase cDNA (Tr). Conditioned medium (Med) and cell lysates were analyzed by immunoblotting for TFPI expression and extracellular accumulation. C) HEK-293 cells were transfected with wild type latent $65 \mathrm{kDa}$ heparanase (65) or heparanase gene construct lacking its heparin-binding domain $(65 \Delta 10)$. Conditioned medium and cell lysates were subjected to immunoblotting, as described above. D) U87 cells were left untreated as control (Con), or pretreated with bacterial heparinase II $\left(0.01\right.$ unit $\left./ \mathrm{ml}, \mathrm{Ih}, 37^{\circ} \mathrm{C}\right)$. The culture medium was then collected and analyzed by immunoblotting utilizing anti-TFPI antibodies.

eluted material was analyzed by immunoblotting using monoclonal anti-heparanase antibody. Similarly, serum free medium of control mock transfected (Con) and heparanase transfected (Hepa) U87 cells were incubated with immobilized polyclonal anti-heparanase antibody and the eluted material was analyzed for the presence of TFPI, applying polyclonal anti-TFPI antibody. As demonstrated in Figure 4E, heparanase and TFPI form a stable complex that can be readily detected by co-IP. Similar results were obtained by co-IP of purified GS3 active heparanase $(0.5 \mu \mathrm{g})$ and recombinant TFPI $(0.5 \mu \mathrm{g})$ proteins (Fig. $4 \mathrm{~F})$. Controls resulted in little or no signal in the above described co-IP experiments (Fig. 4F). Thus, it appears that heparanase exerts early
Heparinase II pretreated and control cells were washed twice with serum free medium and then incubated for $\mathrm{I} h$ with active heparanase $(\mathrm{l} \mu \mathrm{g} / \mathrm{ml})$. Culture medium (second panel) and cell lysates (third panel) were subjected to immunoblotting with anti-TFPI and anti-actin (lower panel) antibodies. E) Co-IP. Overnight incubated serum free media of control mock transfected (Con) and heparanase transfected (Hepa) U87 cells $\left(\mathrm{I} \times 10^{6} / \mathrm{ml}\right)$ were incubated with beads coupled to polyclonal anti-heparanase (upper panel), or anti-TFPI (lower panel) antibodies. Bound proteins were analyzed by immunoblotting for the presence of TFPI (upper, left) or heparanase (lower, left), using the respective antibody, as described in Materials and methods. F) Similarly, purified TFPI and GS3 active heparanase proteins were incubated (T), and co-IP experiment was carried out as described above. Note the interaction between TFPI and heparanase, and the absence of interaction with beads coupled to irrelevant anti-GST polyclonal antibody (Con-I), or uncoupled beads (Con-2).

and late effects on TFPI. At an early time point (i.e. $30 \mathrm{~min}$ ) it enhances extracellular accumulation of TFPI by forming a stable complex that dissociates TFPI from the plasma membrane, while at a later time point (i.e. $6 \mathrm{~h}$ ) it induces TFPI gene transcription.

\section{Discussion}

TFPI is a plasma Kunitz-type serine protease inhibitor and is the only known endogenous modulator of blood coagulation initiated by TF (24). Here, we demonstrate that exogenous addition or over expression of heparanase by transfected cells results in the release of TFPI from the cell surface and its accumulation in 
the cell culture medium. TFPI released into the medium was inactive (Fig. 1), likely due to its interaction with heparanase, further supporting the procoagulant effect of heparanase. Importantly, in-vitro studies are supported by the elevation of TFPI levels in the plasma of transgenic mice over-expressing heparanase (Fig. 2). Moreover, increased levels of TFPI have been noted in the plasma of cancer patients $(25,26)$, possibly reflecting the induction of heparanase expression and elevation of its plasma levels revealed by a newly developed ELISA assay (27) (Shafat et al., data not shown). In HUVEC and tumor derived cell lines, release of TFPI from the cell surface correlated with enhanced TFmediated coagulation (Fig. 3). The fact that accumulation of TFPI in the medium and increased procoagulant activity on the cell surface were detected already within $1 \mathrm{~h}$ of incubation of HUVEC with heparanase, prior to the induction of TF [14] or TFPI, indicates that the release of TFPI is of the mature, preformed protein rather than a newly synthesized TFPI (Figs. 1E, $3 \mathrm{~A})$. In parallel, after $6 \mathrm{~h}$ of incubation with heparanase, induction of TFPI was observed at both the mRNA and protein levels (Fig. 1E, G). Thus, heparanase enhances local coagulation activity via two independent mechanisms with different kinetics, namely, TFPI dissociation from the cell surface followed by induction of TF expression (14). Both functions require secretion of heparanase, but no enzymatic activity, yet the underlying mechanism is apparently different. While TF induction appears to be mediated by the p38 signaling pathway via a putative heparanase binding protein/receptor (14), the release of TFPI is likely due to its physical interaction with the secreted heparanase, as is clearly evident by co-IP experiments (Fig. 4), reflecting for the first time a functional interaction between heparanase and a membrane protein. Extracellular accumulation of TFPI upon heparanase addition suggests that following their interaction, the complex TFPI/heparanase dissociates from the plasma membrane and accumulates extracellularly.

Elevated levels of heparanase may be generated locally upon degranulation of neutrophils, mast cells and platelets (28), further facilitating blood coagulation at the site of platelet activation. Extracellular heparanase is also upregulated upon cell transformation and cancer progression (Shafat et al., data not shown). Heparanase upregulation is noted in essentially all primary human tumors examined, correlating with reduced post op- erative survival and poor prognosis (3-10). Cancer patients often display a pro-thrombotic state due to the ability of tumor cells to activate the coagulation system. Over-expression of TF and acquired activated protein C resistance (APC resistance) were suggested to be the main factors for coagulopathy conditions in malignant disorders (29). Hemostatic function of heparanase, executed by inducing TF expression and releasing TFPI from the endothelial cell surface, provides a mechanism by which heparanase contributes to tumor complication, in addition to its established pro-angiogenic and pro-metastatic activities $(1,2)$.

Low TFPI was found to be a risk factor for venous thrombosis (30) while treatment with heparins increases the level of TFPI in plasma, further enhancing the systemic anticoagulant effect of heparins (31). In addition, species of heparins which do not affect clotting time, still release TFPI and exert an anti-metastatic effect (32-34). Heparanase is strongly inhibited by heparins, regardless of their effect on clotting time (35). Inhibition of heparanase by heparin may reduce the release of heparanase-bound TFPI. Moreover, heparins enable the release of active TFPI, thus potentially neutralizing, in part, the pro-coagulant effect of heparanase. This course of events may further contribute to the anti-coagulant effect of heparins. Taken together, our results support the notion that heparanase is a modulator of blood coagulation, and suggest a novel mechanism by which heparanase regulates TFPI levels on the surface of endothelial and cancer cells. The elevation of heparanase levels in human tumors together with the pro-thrombotic state of most neoplasms, suggest a possible clinical relevance of the procoagulant function of heparanase, as was demonstrated for leukemia patients (14). Targeting domains of heparanase that mediate its enzymatic activity-dependent and independent functions may prove beneficial for patients with cancer and pro-thrombotic conditions.

\section{Acknowledgements}

We are grateful to InSight Biopharmaceuticals (Rehovot, Israel) for providing the anti-heparanase monoclonal antibody (Mab 130). This work was supported by the Aventis/ISTH research fellowship award and by grants from the Israel Science Foundation (grant 549/06); National Cancer Institute, NIH (grant RO1-CA106456); the Israel Cancer Research Fund; and the Rappaport Family Institute Fund.

\section{References}

1. Parish CR, Freeman C, Hulett MD. Heparanase: a key enzyme involved in cell invasion. Biochim Biophys Acta 2001; 1471: M99-108.

2. Vlodavsky I, Abboud-Jarrous G, Elkin M, et al. The impact of heparanese and heparin on cancer metastasis and angiogenesis. Pathophysiol Haemost Thromb 2006; 35: 116-127.

3. Bar-Sela G, Kaplan-Cohen V, Ilan N, et al. Heparanase expression in nasopharyngeal carcinoma inversely correlates with patient survival. Histopathology 2006; 49: 188-193.

4. Koliopanos A, Friess H, Kleeff J, et al. Heparanase expression in primary and metastatic pancreatic cancer. Cancer Res 2001; 61: 4655-4659.

5. Rohloff J, Zinke J, Schoppmeyer K, et al. Heparanase expression is a prognostic indicator for postoper- ative survival in pancreatic adenocarcinoma. $\mathrm{Br} \mathrm{J}$ Cancer 2002; 86: 1270-1275.

6. Gohji K, Hirano H, Okamoto M, et al. Expression of three extracellular matrix degradative enzymes in bladder cancer. Int J Cancer 2001; 95: 295-301.

7. Takaoka M, Naomoto Y, Ohkawa T, et al. Heparanase expression correlates with invasion and poor prognosis in gastric cancers. Lab Invest 2003; 83: 613-622.

8. Tang W, Nakamura Y, Tsujimoto M, et al. Heparanase: a key enzyme in invasion and metastasis of gastric carcinoma. Mod Pathol 2002; 15: 593-598.

9. Shinyo Y, Kodama J, Hongo A, et al. Heparanase expression is an independent prognostic factor in patients with invasive cervical cancer. Ann Oncol 2003; 14: $1505-1510$.
10. Sato T, Yamaguchi A, Goi T, et al. Heparanase expression in human colorectal cancer and its relationship to tumor angiogenesis, hematogenous metastasis, and prognosis. J Surg Oncol 2004; 87: 174-181.

11. Gingis-Velitski S, Zetser A, Flugelman MY, et al. Heparanase Induces Endothelial Cell Migration via Protein Kinase B/Akt Activation. J Biol Chem 2004; 279: 23536-23541.

12. Zetser A, Bashenko Y, Edovitsky E, et al. Heparanase induces vascular endothelial growth factor expression: correlation with p38 phosphorylation levels and Src activation. Cancer Res 2006; 66: 1455-1463. 13. Elkin M, Ilan N, Ishai-Michaeli R, et al. Heparanase as mediator of angiogenesis: mode of action. Faseb J 2001; 15: 1661-1663. 
14. Nadir Y, Brenner B, Zetser A, et al. Heparanase induces tissue factor expression in vascular endothelial and cancer cells. J Thromb Haemost 2006; 4: 2443-2451.

15. Gingis-Velitski S, Zetser A, Kaplan V, et al. Heparanase Uptake Is Mediated by Cell Membrane Heparan Sulfate Proteoglycans. J Biol Chem 2004; 279: 44084-44092.

16. Zetser A, Bashenko Y, Miao H-Q, et al. Heparanase Affects Adhesive and Tumorigenic Potential of Human Glioma Cells. Cancer Res 2003; 63: 7733-7741.

17. Zetser A, Levy-Adam F, Kaplan V, et al. Processing and activation of latent heparanase occurs in lysosomes. J Cell Sci 2004; 117: 2249-2258.

18. Hulett MD, Hornby JR, Ohms SJ, et al. Identification of active-site residues of the pro-metastatic endoglycosidase heparanase. Biochemistry 2000; 39: 15659-15667.

19. Levy-Adam F, Abboud-Jarrous G, Guerrini M, et al. Identification and characterization of heparin/heparan sulfate binding domains of the endoglycosidase heparanase. J Biol Chem 2005; 280: 20457-20466.

20. Nardella C, Lahm A, Pallaoro M, et al. Mechanism of activation of human heparanase investigated by protein engineering. Biochemistry 2004; 43: 1862-1873. 21. Zcharia E, Metzger S, Chajek-ShaulL T, et al. Transgenic expression of mammalian heparanase uncovers physiological functions of heparan sulfate in tis- sue morphogenesis, vascularization, and feeding behavior. FASEB J 2004; 18: 252-263.

22. Ho G, Broze GJ Jr, Schwartz AL. Role of heparan sulfate proteoglycans in the uptake and degradation of tissue factor pathway inhibitor-coagulation factor Xa complexes. J Biol Chem 1997; 16838-16844.

23. Fuki II, Iozzo RV, Williams KJ. Perlecan heparan sulfate proteoglycan. A novel receptor that mediates a distinct pathway for ligand catabolism. J Biol Chem 2000; 275: 25742-50.

24. Lwaleed BA, Bass PS. Tissue factor pathway inhibitor: structure, biology and involvement in disease. Pathol 2006; 208: 327-339.

25. Iversen N, Lindahl AK, Abildgaard U. Elevated TFPI in malignant disease: relation to cancer type an hypercoagulation. Br J Haematol 1998, 102: 889-895. 26. Gerlach R, Scheuer T, Bohm M, et al. Increased levels of plasma tissue factor pathway inhibitor in patients with glioblastoma and intracerebral metastases. Neuro Res 2003; 25: 335-338.

27. Shafat I, Zcharia E, Nisman B, et al. An ELISA method for the detection and quantification of human heparanase. Biochem Biophys Res Commun 2006; 341: 958-963.

28. Vlodavsky I, Eldor A, Haimovitz-Friedman A, et al. Expression of heparanase by platelets and circulating cells of the immune system: possible involvement in diapedesis and extravasation. Invasion Metastasis 1992; 12: 112-127.
29. Haim N, Lanir N, Hoffman R, et al. Acquired activated protein $\mathrm{C}$ resistance is common in cancer patients and is associated with venous thromboembolism. Am J Med 2001; 110: 91-96.

30. Hoke M, Kyrle PA, Minar E, et al. Tissue factor pathway inhibitor and the risk of recurrent venous thromboembolism. Thromb Haemost 2005; 94: 787-790.

31. Sarig G, Blumenfeld Z, Leiba R, et al. Modulation of systemic hemostatic parameters by enoxaparin during gestation in women with thrombophilia and pregnancy loss. Thromb Haemost 2005; 94: 980-985.

32. Mousa SA, Linhardt R, Francis JL, et al. A. Antimetastatic effect of a non-anticoagulant low-molecular-weight heparin versus the standard low-molecularweight heparin, enoxaparin. Thromb Haemost 2006; 96:816-21.

33. Hostettler N, Naggi A, Torri G, et al. P-selectin- and heparanase-dependent antimetastatic activity of nonanticoagulant heparins. FASEB J 2007; 21: 3562-3572.

34. Liu T, Scallan CD, Broze GJ Jr, et al. Improved coagulation in bleeding disorders by Non-Anticoagulant Sulfated Polysaccharides (NASP). Thromb Haemost 2006; 95: 68-76.

35. Naggi A, Casu B, Perez M, et al. Modulation of the heparanase-inhibiting activity of heparin through selective desulfation, graded N-acetylation, and glycol splitting. J Biol Chem 2005; 280: 12103-12113. 